\title{
Implementation of Scaling and Extended Scaling Equations of State for the Critical Point of Fluids
}

\author{
M. R. Moldover \\ National Measurement Laboratory, National Bureau of Standards, Washington, D. C. 20234 \\ February 21, 1978

\begin{abstract}
An explicit, practical procedure is suggested for transforming from the laboratory variables density $(\rho)$ and temperature $(T)$ into the parametric variables $r$ and $\theta$, which occur in various scaled representations of equations of state and of transport properties of fluids near critical points. A reasonably efficient and versatile computer program illustrating this procedure is provided. With this program, the parametric equations of state which occur in several formulations of simple, extended, and/or revised scaling are as easy to use as any other equation of state for which $T$ and $\rho$ are the independent variables.
\end{abstract}

Key words: Critical point; equation of state; scaling equation of state.

\section{Introduction}

In recent years various scaling equations of state have been developed to provide theoretically based, highly accurate representations of thermodynamic data for pure fluids and fluid mixtures near their critical points. These equations of state are all consistent with the hypothesis that very near the critical point the thermodynamic potential is a homogeneous function of its natural variables. These equations of state have relatively few parameters which must be adjusted to fit data for any specific fluid. This is a practical advantage for those concerned with taking or correlating data. On the other hand, many scaling equations of state are written in terms of parametric variables which appear in nonlinear equations linking together such physically relevant variables as the pressure $(P)$, the density $(\rho)$, and the temperature $(T)$. The occurrence of the parametric variables is a major practical disadvantage to any nonexpert who might otherwise be interested in using a scaling equation of state to deal with a limited problem. It is, of course, true that these parametric variables can be eliminated algebraically from the equation of state along special paths in thermodynamic space such as the vapor pressure curve. Then, along the special paths, the scaling equations reduce to simple power laws with nonintegral exponents relating directly measured quantities to each other. For states off these special paths, a user of a scaling equation of state must eliminate the parametric variables numerically. The purpose of the present paper is to provide an explicit example of one way in which this can be done. If our method is followed, a user need not repeat the programming effort required to eliminate the parametric variables in

\footnotetext{
${ }^{1}$ Figures in brackets indicate literature references at the end of the paper.
}

those situations in which $\rho$ and $T$ are independent variables. In situtations in which it is convenient to designate other physical variables as independent, the general method outlined here may still be followed.

In this work we discuss only those scaling equations of state which use parametric variables. There are several published examples of scaling equations of state which do not use parametric variables $[1,2] .{ }^{1}$ Unfortunately, these non-parametric equations of state cannot be integrated in closed form to obtain an explicit expression for the thermodynamic potential. Thus, frequently used expressions for the entropy and for the specific heats consistent with nonparametric equations of state contain integrals which must be evaluated numerically. In our experience, the lack of an explicit thermodynamic potential has been a serious handicap in attempting to use these non-parametric equations for constructing thermodynamic models for real fluids. Accordingly, we now choose to deal with the numerical problems arising from the use of parametric variables rather than the numerical problems associated with integral representations for the potential.

\section{Simple Scaling with the Linear Model}

We will consider first the "linear model" parametric equation of state proposed by Schofield [3]. This equation was first proposed on phenomenological grounds and was applied with success to the insulating ferromagnet $\mathrm{CrBr}_{3}$ [4]. It has since received some theoretical justification [5] and it has been widely used to correlate equation of state and transport property data in various fluid systems [6-9]. Most important from our present point of view, the equations to be solved in using the linear model parametric equation of state 
may be easily generalized to handle the more complicated cases of extended and/or revised scaling.

To use the linear model parametric equation of state in situations where the temperature $(T)$ and the density $(\rho)$ are the independent variables, the parametric variables $r$ and $\theta$ must be obtained by solving the following two equations:

$$
\begin{aligned}
& T=T_{c}+T_{c} r\left(1-b^{2} \theta^{2}\right) \\
& \rho=\rho_{c}+\rho_{c} m r^{\beta} \theta
\end{aligned}
$$

Here $T_{c}$ and $\rho_{c}$ are critical constants. For the simple fluids studied by Levelt Sengers et al., [7] $m$ is a positive constant in the range $1.5<m<2.1 ; b^{2}$ is a constant falling in the range $1.1<b^{2}<1.6$, and the exponent $\beta$ usually falls in the range $0.31<\beta<0.38$. The transformation represented by eqs (1) and (2) for the fluid xenon is sketched in figure 1 . To make this sketch, values of $T_{c}, \rho_{c}, m$, and $b^{2}$ were taken from reference [7]. Once values of $r$ and $\theta$ are obtained by solving (1) and (2), other quantities such as the pressure $(P)$ and the constant volume specific heat $\left(C_{v}\right)$ may be calculated as functions of $\rho$ and $T$ by simply substituting $r$ and $\theta$ into algebraic equations which can be solved explicitly for the desired quantities $[7,10]$. There are many ways to solve these equations numerically. The method described here is reasonably efficient and reliable.

To solve (1) and (2) we first introduce the dimensionless variables $\Delta T^{*}=\left(T-T_{c}\right) / T_{c}$, and $\Delta \rho^{*}=\left(\rho-\rho_{c}\right) / \rho_{c}$ to obtain

$$
\begin{aligned}
& 0=\Delta T^{*}-r\left(1-b^{2} \theta^{2}\right) \\
& 0=\Delta \rho^{*}-m r^{\beta} \theta
\end{aligned}
$$

We then eliminate $r$ from (1) and (2) to obtain

$$
0=-\Delta \rho^{*} \frac{b}{m}\left|\Delta T^{*}\right|^{-\beta}+b \theta\left|1-b^{2} \theta^{2}\right|^{-\beta}
$$

Note that absolute value signs had to be introduced because we have chosen to raise to powers the separate quantities $\Delta T^{*}$ and $\left(1-b^{2} \theta^{2}\right)$ rather than their quotient which always remains positive. By introducing two new symbols, $Z$ and $C$

$$
\begin{aligned}
& Z=b \theta \\
& C=-\Delta \rho^{*} \frac{b}{m}\left|\Delta T^{*}\right|^{-\beta}
\end{aligned}
$$

we obtain a compactly written transcendental equation in one variable

$$
f(Z)=0=C+Z\left|1-Z^{2}\right|^{-\beta}
$$

Numerical approximations to its roots may be found efficiently by Newton's method. This is particularly easy to implement because the derivative, $f^{\prime}(Z)$, may be calculated analytically. In Newton's method, the $(n+1)$ 'th estimate for $Z$ is found from the $n^{\prime}$ th estimate by the rule

$$
Z_{n+1}=Z_{n}-\frac{f(Z)}{f^{\prime}(Z)}
$$

In the present case this rule takes the form

$$
Z_{n+1}=Z_{n}-\frac{\left(1-Z_{n}^{2}\right)\left(Z_{n}+C_{n}\left|1-Z_{n}^{2}\right|^{\beta}\right)}{\left(1-Z_{n}^{2}\right)+2 \beta Z_{n}^{2}}
$$

Equation (9) is quite satisfactory for computation if suitable precautions are taken to handle the singularity at $Z=1$. Each iteration requires only a single exponentiation. A reasonable initial estimate for $Z$ is obtained by looking at the

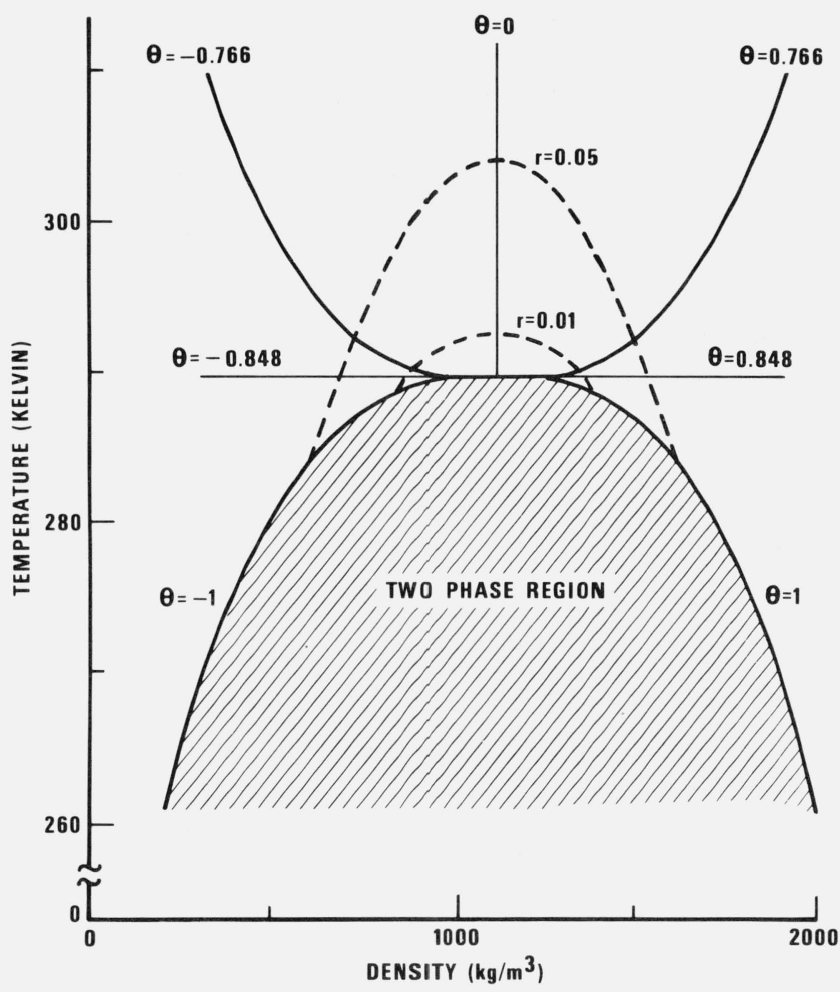

Figure 1. Sketch of the transformation from $\rho$ and $T$ to $r$ and $\theta$ in the linear model (eqs (1) and (2)) with parameters appropriate for xenon from reference [7].

physically important special curves, namely: the critical isochore above $T_{c}$ for which $Z=0$, the critical isotherm for which $Z= \pm 1$ and finally or coexistence curve on which $Z$ $=+b$ in the liquid phase and $Z=-b$ in the vapor phase. An estimate which has the appropriate limits near the special curves is: 


$$
\begin{aligned}
& Z_{0}=1-\frac{(1-b) \Delta T^{*}}{1-b^{2}}\left|\frac{m}{\Delta \rho^{*}}\right|^{1 / \beta} \text { for } \Delta T^{*}<0 \\
& Z_{o}=\left(1+\Delta T^{*}\left|\frac{m}{b \Delta \rho^{*}}\right|^{1 / \beta}\right)^{-\beta} \text { for } \Delta T^{*}>0
\end{aligned}
$$

We have applied this approach to the solution of eqs (3) and (4) with the double precision Fortran program listed in appendix I. For all of the physically reasonable values of input parameters we have tried, at most five iterations were needed. The resulting values of $r$ and $\theta$ satisfy eqs (3) and (4) to 12 or more significant figures. (When $\left|\Delta \rho^{*}\right|$ and/or $\left|\Delta T^{*}\right|$ are smaller than $10^{-12}$, an "unphysical" situation, the sample program yields fewer significant figures). If a substantial amount of computation were to be done with a single value of $\beta$, a program could be written in which the timeconsuming step of raising a floating point number to a floating point power occurs less frequently than the $2-7$ times it occurs with the present program.

In concluding this section we note that solutions to (3) and (4) in the range $1<\theta^{2}<1 /\left[b^{2}(1-2 \beta)\right]$ may be interpreted as either two phase states (for which $\theta$ should be set to \pm 1 ) or metastable extensions of the single phase isotherms [11]. Very little is known about the accuracy of the linear model when it is used in the metastable region.

\section{Additional Examples Using Simple Scaling}

Other familiar parametric versions of simple scaling can be handled in the same way as the "linear model". For example, the "cubic model" introduced by Ho and Litster [12] uses two parametric variables which may be considered to be defined by the equations:

$$
\begin{aligned}
& 0=\Delta T^{*}-r\left(1-b^{2} \theta^{2}\right) \\
& 0=\Delta \rho^{*}-m r^{\beta} \theta\left(1+c_{2} \theta^{2}\right)
\end{aligned}
$$

Again we have used $r$ and $\theta$ as the parametric variables while $m, b, \beta$, and $c_{2}$ are constants. If the same substitutions are made in (11) and (12) as those used above in connection with the linear model, one may obtain:

$$
f(Z)=0=C+Z\left(1+\frac{c_{2}}{b^{2}} Z^{2}\right)\left|1-Z^{2}\right|^{-\beta}
$$

In practice, $c_{2} Z / b^{2}$ is much less than unity so that (13) is very similar to (7). Initial estimates for the solution to (13) may be obtained from eq (10), just as for the "linear model"; then the solution by Newton's method proceeds exactly as outlined above except that the "cubic model" function for $f(Z)$ is used instead of the linear model function.
A parametric scaling equation of state was introduced by Wilcox and Estler [13] to deal with data from diffraction experiments in fluids near critical points. Their scheme has been used by Estler et al. [14] and by Hocken and Moldover [15]. For a given fluid state, the parametric variables in the Wilcox-Estler scheme have numerical values which are quite different from those which appear in the "linear model" or the "cubic model". One may consider the variables to be defined by the equations:

$$
\begin{gathered}
0=\left|\Delta \rho^{*}\right|-\left[Y_{0} R\left(1-\theta / \theta_{0}\right)^{\Delta}\right]^{\beta} \\
\times\left[1+\frac{\beta}{\gamma} \frac{1-\theta / \theta_{x}}{1-\theta / \theta_{0}}\right] \\
0=\Delta T^{*}-R \theta
\end{gathered}
$$

Here, following Wilcox and Estler, the parametric variables are " $R$ " and " $\theta$ " while $Y_{o}, \theta_{o}, \theta_{x}, \beta$ and $\gamma$ are all constants, and $\Delta$ is a combination of constants: $\Delta=1-\theta_{o} / \theta_{x}$. (Note: Because the absolute value of $\Delta \rho^{*}$ appears in (14), the inverse transformation from $R$ and $\theta$ to $\Delta \rho^{*}$ and $\Delta T^{*}$ is not unique). Once again one of the parametric variables, say $R$, may be eliminated from (14) and (15) to yield an equation in one variable which may be solved by Newton's method.

On occasion, $T$ and $\rho$ are not the most convenient independent variables. For example, the analysis of flow calorimetry data [16] requires the computation of the enthalpy as a function of $T$ and $P$. Each of the simple scaling equations of state mentioned above will lead to two equations relating $P$ and $T$ to parametric variables. The numerical problem of solving these equations for $r$ and $\theta$ is quite similar to the problem we have discussed above, namely of solving the equations in $\rho$ and $T$ for $r$ and $\theta$. In particular, when $P$ and $T$ are known it is still possible to eliminate one of the parametric variables from the two equations algebraically. Thus a single equation in one unknown remains which may be efficiently solved with Newton's method.

\section{Extended and/or Revised Scaling}

In more complicated versions of scaling, the conversion from the laboratory variables $\rho$ and $T$ to the parametric variables $r$ and $\theta$ requires the numerical solution of two simultaneous equations. One parameter can no longer be eliminated algebraically. An efficient and versatile approach is to use simple scaling to obtain a first approximation to the values of the parametric variables. The approximation may then be improved by using Newton's method for the solution of simultaneous equations.

The same first approximation (simple scaling) may be used with a variety of more complicated models. Where the derivatives required for the use of Newton's method are computed numerically, it becomes quite easy to change from one model to another. 
We illustrate this approach by considering the form of extended and revised scaling used by Balfour et al. [10] to study the equation of state of steam near its critical point. In this scheme the defining equations may be written in a form analogous to eqs (3) and (4) above:

$$
\begin{aligned}
U(r, \theta)=0= & \Delta T^{*}-r\left(1-b^{2} \theta^{2}\right) \\
& \times\left[1-q a r^{1-\alpha-\beta}\left(\frac{\theta\left(1-\theta^{2}\right.}{1-b^{2} \theta^{2}}\right)\right] \\
V(r, \theta)=0= & \Delta \rho_{d}^{*}-m r^{\beta} \theta \\
& \times\left\{1+\frac{k_{1}{ }^{r \Delta}}{m}+q a r^{1-\alpha-\beta} S_{0}(\theta) / \theta\right. \\
& \left.+q a k_{1} r^{1-\alpha-\beta+\Delta} S_{1}(\theta) /(m \theta)\right\}
\end{aligned}
$$

Here, the variable $\Delta \rho_{d}^{*}$ is the scaled density deviation from the "rectilinear diameter" (In the notation of Balfour et al. $\Delta \rho_{d}^{*}=A_{11}^{*} \Delta T^{*}+\Delta \rho^{*}$ ). This contrasts with $\Delta \rho^{*}$ used above which is the scaled density deviation from the critical density. In (16) and (17) the exponents $\alpha$ and $\Delta$ are about 0.1 and 0.5 , respectively. The constants $q$ and $k_{1} / m$ are small compared with unity, and $S_{0}(\theta)$ and $S_{1}(\theta)$ are rational polynomial functions of $\theta$.

To consider the transformations (16) and (17), we recall that both revised and extended scaling may be cast in the form of an expansion about the critical point. Thus the small "revision" coefficient $q$ is a measure of the lowest order departure of the isotherms from antisymmetry and the small "extension" coefficient $k_{1} / m$ is a measure of the strength of the second most singular term in the free energy expansion about the critical point. We expect that if the expansion is to make sense, the terms with these coefficients should be small compared with the leading terms. Thus, for physically meaningful values of $\Delta \rho_{d}^{*}$ and $\Delta T^{*}$, good initial estimates for the parametric variables $r$ and $\theta$ in revised and/or extended scaling can be found by setting $k_{1} / m$ and $q$ equal to zero. This, of course, reduces eqs (10) and (11) to eqs (3) and (4) which we have already solved in dealing with simple scaling.

Then, the initial estimates for $r$ and $\theta$ may be improved efficiently by using Newton's method for the solution of equations in two variables. The $(n+1)$ 'th estimates for $r$ and $\theta$ are obtained from the $n^{\prime}$ th estimate by the rules [17]

$$
\begin{gathered}
r_{n+1}=r_{n}-\left[U\left(r_{n}, \theta_{n}\right) \frac{\partial V}{\partial \theta}-V\left(r_{n}, \theta_{n}\right) \frac{\partial U}{\partial \theta}\right] / D \\
\theta_{n+1}=\theta_{n}-\left[V\left(r_{n}, \theta_{n}\right) \frac{\partial U}{\partial r}-U\left(r_{n}, \theta_{n}\right) \frac{\partial V}{\partial r}\right] / D \\
D=\frac{\partial U}{\partial r} \frac{\partial V}{\partial \theta}-\frac{\partial U}{\partial \theta} \frac{\partial V}{\partial r}
\end{gathered}
$$

The partial derivatives in eqs (18), (19), and (20) are understood to be evaluated near $\left(r_{n}, \theta_{n}\right)$. In practice we have computed approximations for them from divided differences. Thus, for each iteration, one must evaluate $U$ and $V$ at three sets of coordinates: $(r, \theta),(r+\delta, \theta)$, and, $(r, \theta+\delta)$. The small difference, $\delta$, used for estimating the derivatives is chosen to be $10^{-8}$ in the sample program in the appendix. This value is satisfactory for all the physically reasonable values of $\Delta T^{*}$ and $\Delta \rho_{d}^{*}$ we have examined. The singularity at $\Delta T^{*}=\Delta \rho_{d}^{*}=0$ does not cause numerical problems because, near this point, simple scaling gives quite accurate values of $r$ and $\theta$, and the iteration of eqs (18-20) is not used.

If one wished to alter the scheme of Balfour et al., say by adding an additional extension to scaling term with a new coefficient, $k_{2}$, the only change needed in this computational scheme woud be the addition of that term to the functions $U(r, \theta)$ and $V(r, \theta)$.

It is economical to evaluate $U$ and $V$ in the same subprogram to minimize the number of exponentiations required in each iteration.

\section{References}

[1] Griffiths, R. B., Phys. Rev. 158, 176 (1967).

[2] Vicentini-Missoni, M., Levelt Sengers, J. M. H. and Green, M. S., J. Res. Nat. Bur. Stand. (U.S.), 73A (Phys. and Chem.), No. 6, 563583 (Nov.-Dec. 1969).

[3] Schofield, P., Phys. Rev. Lett. 22, 606 (1969).

[4] Ho, J. T. and Litster, J. D., Phys. Rev. Lett. 22, 603 (1969).

[5] Wallace, D. J., in "Phase Transitions and Critical Phenomena," Vol. 6, C. Domb and M. S. Green, Eds., Academic Press, New York, 1976, pp. 293-356.

[6] Kierstead, H. A., Phys. Rev. A7, 242 (1972).

[7] Levelt Sengers, J. M. H., Greer, W. L. and Sengers, J. V., J. Physical and Chemical Reference Data, 5, 1 (1976).

[8] Moldover, M. R., and Gallagher, J. S. AIChE Journal, 24, 267 (1978).

[9] Basu, R. S., and Sengers, J. V., Proceedings of the 7th Symposium on Thermophysical Properties, Gaithersburg, Md., May 10-12, 1977, ASME. pp. 822-830.

[10] Balfour, F. W., Sengers, J.V., Moldover, M.R., and Levelt Sengers, J. M. H., Proceedings of the 7th Symposium on Thermophysical Properties, Gaithersburg, Md., May 10-12, 1977, ASME. pp. 786-793.

[11] Schofield, P., Litster, J. D. and Ho, J. T., Phys. Rev. Lett. 23, 1098 (1969).

[12] Ho, J. T. and Litster, J. D., Phys. Rev. B2, 4523 (1970).

[13] Wilcox, L., and Estler, W. T., J. de Physique (Paris) 32, C5a-175 (1971).

[14] Estler, W. T., Hocken, R., Charlton, T., and Wilcox, L. R., Phys. Rev. A12, 2118 (1975).

[15] Hocken, R. and Moldover, M. R., Phys. Rev. Lett. 37, 29 (1976),

[16] Hejmadi, A. V., Allen, K., and Powers, J. E., Proceedings of the 7th Symposium on Thermophysical Properties, Gaithersburg, Md., May 10-12, 1977. ASME. pp. 807-813.

[17] Scarborough, J. B., Numberical Mathematical Analysis, (Johns Hopkins Press, Baltimore, Md, 1958), p. 204. 


\section{Appendix I}

The subroutine RTHETA solves eqs (3) and (4) of the text for the parametric variables $r$ and $\theta$. The argument list of the subroutine corresponds to the symbols in those equations in the order: $r, \theta, \Delta \rho^{*}, \Delta T^{*}, \beta, m, b^{2}$. This version treats thermodynamic states for which $1<|\theta|<1.00234$ as though such states were metastable. (The number 1.00234 is arbitrarily chosen. It must fall within the range indicated in the text). States for which $1.00234<|\theta|$ are treated as two phase states. In other words, $\theta$ is assigned the value +1 or -1 which is appropriate for an equilibrium liquid or vapor state at coexistence conditions.

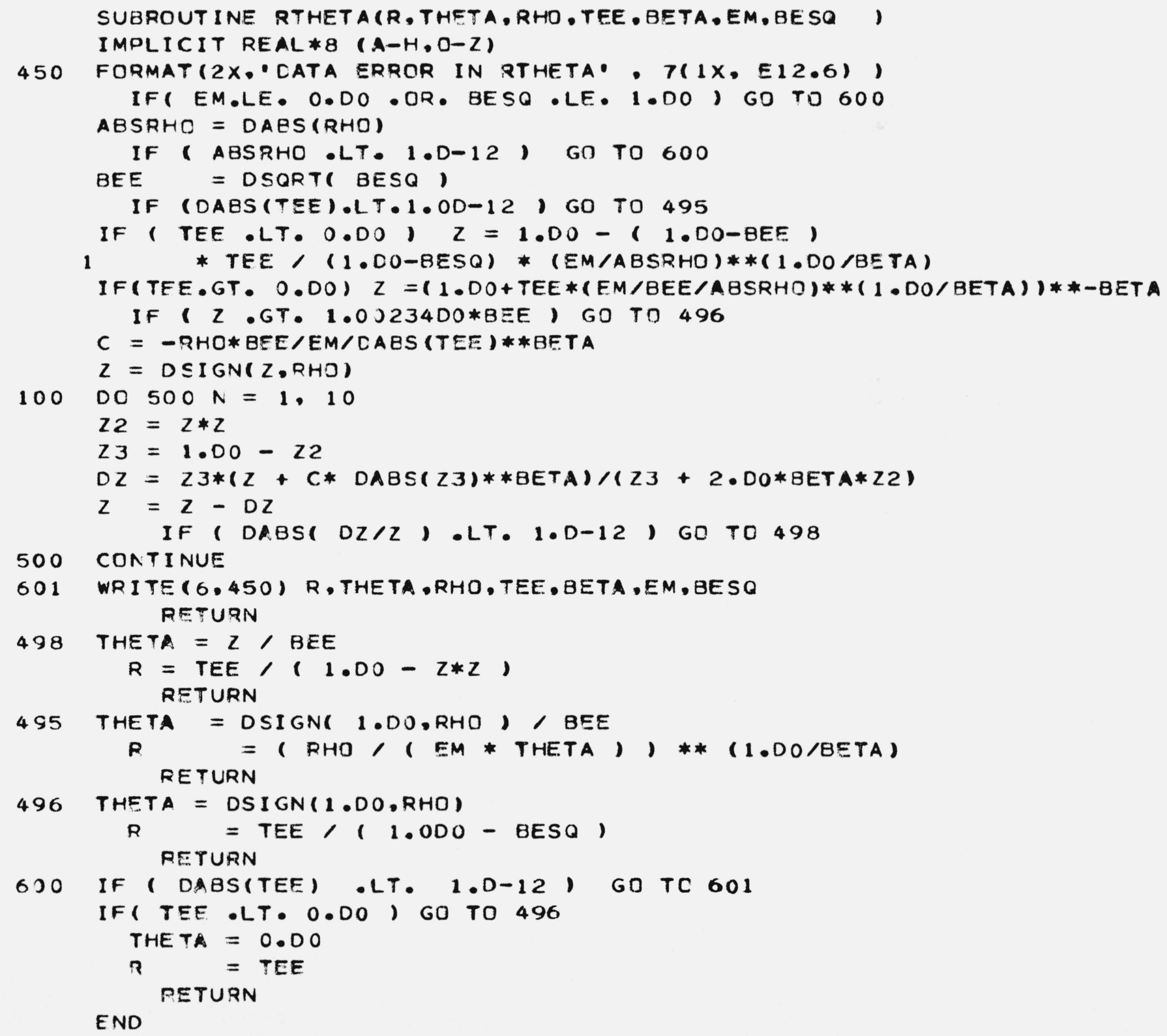




\section{Appendix II}

This subroutine, SOLVE, solves equations similar to (16) and (17) of the text for the parametric variables $r$ and $\theta$, The argument list is identical with that in appendix I. This subroutine assumes that the subroutine RTHETA of appendix I is available and that another subprogram, $U V$, is available to evaluate the functions $U(r, \theta)$ and $V(r, \theta)$ defined by equations such as (16) and (17). The $U V$ subprogram's argument list corresponds to the symbols $r, \theta, U(r, \theta), V(r, \theta)$, $\Delta \rho^{*}, \Delta T^{*}$, as they are used in eqs (16) and (17). Statement 10 of SOLVE insures that $\theta$ will be assigned a value of +1 or -1 if the values of $\Delta \rho^{*}$ and $\Delta T^{*}$ provided are such as to lie within the coexistence curve. Thus this version of SOLVE does not assume the existence of metastable states.

SUBROUTINE SOLVE(R.THETA,RHO,TEE, BETA.EM,BESQ)

IMPLICIT REAL*8 (A-H,O-Z)

IF (TEE.GE. O.DO) GO TO 55

THETA $=$ DSIGN (1.DO.RHO)

$R=T E E /\left(1 . C_{0}-B E S Q\right)$

CALL UV(R,THETA,U,V,RHO,TEE)

10 IF ( (V*RHO) .LE. O.DO ) GO TO 999

55 CALL RTHETA(R,THETA, RHO,TEE,BETA,EM,BESQ)

$D D=1 \cdot D-8$

65 DO $75 I=1.6$

CALL UV(R,THETA,U,V,RHO,TEE)

IF(DABS(V) .LT. 1.D-5.AND. DABS(U) •LT. 1.D-5) GO TO 999

CALL UV (R+CD,THETA,UR, VR, RHO, TEF)

CALL UV(R, THETA+DD,UT, VT, RHO, TEE)

DUDR = UR - U

DUDT $=$ LT $-U$

DVDR = VR - V

DVDT $=V T-V$

$D=D U D R * D V D T$ - DUCT*DVDR

$R=R-C D *(U * D V D T-V * D U D T) / D$

THETA = THETA - CD*(DUDR*V - DVDR*U) $/ D$

75 CONTINUE

998 WRITE $(6.67)$ RHO, TEE, R, THETA, BETA, EM, BESQ

67 FORMAT(2X, SOLVE,DOES NCT CONVERGE・, 7F15.8)

999 CONTINUE

RETURN

END 